\title{
Chemotactic auto-aggregation in the water mould Achlya
}

\author{
Donovan D. Thomas* and April P. Peterson \\ Department of Biological Sciences, University of Windsor, Windsor, Ontario N9B 3P4, Canada
}

(Received 21 September 1989; revised 11 January 1990; accepted 26 January 1990)

\begin{abstract}
Free-swimming zoospores of several isolates of the oomycetous water mould Achlya demonstrate mutual attraction (adelphotaxis) which may result in auto-aggregation. In the apparent absence of exogenous chemotactic signals macroscopic globular aggregates or microscopic plaques of cysts are formed. Auto-aggregation was favoured by high concentrations of zoospores in suspension; the growth rate of spontaneous aggregates was estimated at 4-10 $\times 10^{3}$ spores min $^{-1}$; growth of an aggregate was limited to about $7 \mathrm{~min}$. By means of chemotactic assays, attractant activity for zoospores was detected in the vicinity of aggregates, and in zoospore or cyst supernatants. The attractant activity extracted from spore aggregates was heat-stable. In the absence of exogenous nutrients, the macroscopic aggregates of an homothallic isolate produced sporangia and oogonia. Aggregation was readily initiated as a result of zoospore chemotaxis towards capillary tubes containing exogenous attractant in an agar medium. Aggregating zoospores frequently assumed a slender swarm configuration moving directly towards the aggregate at the capillary-tube tip. Analysis of video recordings showed that many zoospores joining the swarm were attracted directly by the swarm rather than by the exogenous attractant. Such aggregation was a time-limited process, the duration of which was extended by the presence of amino acids in the zoospore suspension. Under natural conditions zoospore aggregation may be a dual-component chemotactic process triggered by exogenous attractants and amplified by chemical signals from the aggregating spores. The possible consequences of adelphotaxis and auto-aggregate formation, namely enhanced inoculum potential, syngamy and spore production, are discussed.
\end{abstract}

\section{Introduction}

The number of fungal spores present in a group can affect their germination and early development as a result of diffusible regulator molecules (Jaffe, 1966; Robinson, 1973). Enhanced colonization in the presence of increased spore numbers has been reported in plant pathogens with a zoosporic phase (MacWithey, 1965; Kraft \& Endo, 1966). The formation of spore aggregates by free-swimming water mould zoospores was examined in this study.

Although oomycetous zoospores are often considered to be agents of dispersal, they can aggregate as a result of chemotaxis towards an exogenous attractant (Hickman \& Ho, 1966). An example is zoospore chemotaxis to host plant roots (Zentmyer, 1961). In the absence of exogenous chemical gradients, high concentrations of Phytophthora spores have been shown to form loose 'aggregates' of motile spores (Ko \& Chase, 1973). Such 'aggregates' are an example of pattern swimming (Carlile, 1983) which has been interpreted as a bioconvection process (Plessett et al., 1975). The type of zoospore aggregation, auto-aggregation, which we de- scribe results in dense coherent masses of encysted spores. Such aggregation has been reported previously in Saprolegnia (Lechmere, 1910), Isoachlya (Lounsbury, 1927) and Phytophthora (Porter \& Shaw, 1978). Lounsbury (1927) applied the term 'adelphotaxy' (Gk adelphos, brother) to the behaviour of free-swimming zoospores which resulted in the formation of cyst aggregates. The term had been coined by Hartog (1888) to describe mutual cellular attraction resulting in auto-aggregation of a variety of organisms. Phytophthora zoospores aggregate and encyst in clusters in response to what Porter \& Shaw (1978) suggested to be a chemotactic stimulus. We describe here the formation of cyst aggregates as a result of adelphotaxis in Achlya zoospore suspensions and suggest that zoospore chemotaxis to an exogenous attractant may possess an adelphotactic component involving chemical signals from aggregating spores.

\section{Methods}

Cysts of Achlya isolates (ATCC 52875, 52865 and 52866) were prepared as described previously (Thomas, 1989). Unless otherwise specified, the homothallic Achlya heterosexualis isolate, ATCC 52875, 
was used. For each experiment four to six $100 \mathrm{ml}$ cultures were used. Cyst numbers were counted in samples transferred in triplicate to a microscope slide using a $2 \mu \mathrm{l}$ Drummond microcapillary pipette (Ko et al., 1973). The cysts were then concentrated and washed by centrifuging at 2250 r.p.m. for $4 \mathrm{~min}$. After three washes in $10^{-3} \mathrm{M}-$ MOPS [3-( $N$-morpholino)propanesulphonic acid] buffer with $10^{-5} \mathrm{M}$ $\mathrm{CaCl}_{2}$, pH 6.6 (CaMOPS), the spores were resuspended in the buffer and incubated at room temperature until the zoospores emerged.

A calibrated ocular micrometer was used to monitor growth rates of aggregates. In estimating the cyst numbers in a globular aggregate, it was assumed that cysts, of mean diameter $12.6 \mu \mathrm{m}$, were loosely packed, occupying half the volume of the aggregate.

The effect of spore concentration on aggregation was examined by dispensing suspensions of freshly prepared cysts in $0.25 \mathrm{ml}$ samples to Falcon 96-well tissue culture plates with $0.3 \mathrm{ml}$ wells. Spore aggregates were counted and measured after $4 \mathrm{~h}$.

For chemotaxis tests, the rim of a $12 \mathrm{~mm}$ test-tube dipped lightly in silicone grease was used to demarcate two circular dams on a microscope slide. Zoospore suspension $(80 \mu \mathrm{l})$ was gently pipetted into each dam. Attractants tested included spore extracts and various concentrations of L-methionine, previously shown to be chemotropically active in Achlya (Musgrave et al., 1977; Manavathu \& Thomas, 1985). After loading a $2 \mu$ l capillary pipette with a test attractant, the upper end of the tube was sealed with silicone grease. The pipettes with test attractant or control buffer solutions were rested horizontally on a microscope slide with their open ends each centred in a zoospore dam and checked microscopically for air bubbles near the pipette tip. Chemotaxis was terminated after $1-5 \mathrm{~min}$ by sealing the open ends of the tubes with silicone grease; spores trapped in the pipettes were counted. If the number of spores was too large (over 50) for convenient counting, the zoospore suspension was diluted or the trapping time decreased. For each attractant tested a mean of six readings was obtained and three independent replicates were used.

To test for zoospore attractant activity associated with various spore preparations, cysts washed three times and resuspended in CaMOPS buffer to a final concentration of over $5 \times 10^{5}$ spores $\mathrm{ml}^{-1}$ were placed in $3 \mathrm{ml}$ samples in $35 \mathrm{~mm}$ diameter Petri dishes, and spore extracts prepared as described below and in Table 2. The extracts from 8-12 spontaneously initiated aggregates were collected as follows. The upper end of a $20 \mu \mathrm{l}$ capillary pipette was temporarily sealed and the lower end placed above the aggregate; removal of the seal permitted loading with aggregate and suspension fluid. A rubber bulb with an adaptor was used to expel the contents of the pipette into $0.1 \mathrm{ml}$ CaMOPS buffer. After centrifuging for $2 \mathrm{~min}$ in an Eppendorf centrifuge at 14000 r.p.m. the supernatant was tested for chemotactic activity. Zoospore filtrates were obtained using pre-rinsed Millipore filters with a pore size of $3 \mu \mathrm{m}$.

In attempts to nucleate aggregation using chemotactic attractants, $2 \mu \mathrm{l}$ capillary tubes were loaded by stabbing deeply three or four times into a peptone/yeast extract/glucose (PYG; Cantino \& Lovett, 1960) agar plate or a $10^{-3} \mathrm{M}$-methionine agar plate. The tubes containing cores of attractant agar were wiped with tissue paper and immersed in a zoospore suspension. Growth rates of aggregates nucleated by PYG agar in $3 \mathrm{ml}$ of zoospore suspension in a $35 \mathrm{~mm}$ Petri dish were monitored microscopically. In order to explore the possibility that the duration of aggregation might be related to saturation of receptor sites we examined the effect of different medium concentrations of PYG, Lmethionine or L-alanine on aggregation induced by PYG agar.

Polaroid type 667 film was used for photomicrography. Aggregating zoospores were recorded using a $4 \times$ objective of a Kyowa Medilux 12 trinocular microscope, a Panasonic WV-1500 television camera and a Panasonic AG-6050 time-lapse video cassette recorder. Randomly selected zoospores were tracked using tracings from the monitor onto acetate transparencies. Each spore was tracked at 10 -frame $(0.17 \mathrm{~s})$ intervals for up to $3 \mathrm{~s}$.

All experiments were repeated two or more times.

\section{Results}

In suspensions with high spore concentrations $\left(10^{5}-10^{6}\right.$ $\mathrm{ml}^{-1}$ ) all three Achlya isolates tested produced spontaneous aggregates within 2-3 h. Aggregates were coherent masses of encysted zoospores which, unlike the loose zoospore 'aggregates' described by Ko \& Chase (1973), persisted throughout the maximum observation period of a week. The diameters of fully developed aggregates ranged from 1-2 $\mathrm{mm}$ in macroscopic globules to $50-300$ $\mu \mathrm{m}$ in microscopic plaques (Fig. $1 a, b$ ). Macroscopic aggregates formed preferentially on the vertical wall of a container, immediately below the surface of the spore suspension; in an $8.5 \mathrm{~cm}$ diameter Petri plate with an initial concentration of $2.5 \times 10^{5}$ spores $\mathrm{ml}^{-1}, 28$ macroscopic auto-aggregates formed, of which $71 \%$ were on the vertical wall of the plate, $18 \%$ free-floating and $11 \%$ on the floor of the plate. Aggregate growth was rapid, with an estimated 4-10 $\times 10^{3}$ spores min $^{-1}$ added to each aggregate, and appeared to be self-limiting, in that most aggregates ceased to attract zoospores after 5-8 min (Table 1). Aggregates were transferred from the walls to the floor of the plates in experiment 1 , and were undisturbed in experiment 2.

The effect of spore concentration on spontaneous aggregate formation is shown in Fig. 2. With progressive dilution of the original cyst suspensions, fewer zoospore aggregates per microtitre well tended to form; at the lowest two concentrations, the numbers of aggregates were significantly lower than in the undiluted control suspensions. In some experiments, the sizes of the aggregates decreased with spore dilution. In these experiments most aggregates were plaques and globules were rare.

Extracts from cysts and zoospores were chemotactically active and heat-stable (Table 2); we referred to this attractant activity as 'adelphotaxin'. Attractant activity was not specific to a particular Achlya isolate; adelphotaxin from ATCC 52875 strongly attracted zoospores from ATCC 52866 (not shown).

Although isolated asexual spores of Achlya can germinate in the absence of exogenous nutrients, the resulting fine hyphae, with a mean diameter of $2.9 \mu \mathrm{m}$, do not produce spores. The large globular aggregates produced hyphae up to $14 \mu \mathrm{m}$ in diameter, and some developed both asexual spores and oogonia (Fig. $1 d, e$ ). Both sporangia and oogonia were smaller than those produced by typical laboratory cultures. As reported by Porter \& Shaw (1978), germ tubes from cysts in the vicinity of an aggregate were positively chemotropic towards the aggregate (Fig. 1c). Royle \& Hickman (1964a) reported that after Pythium zoospores had aggregated in response to plant roots, germ tubes from spores which had encysted in the vicinity of the aggregate grew chemotropically towards it. 

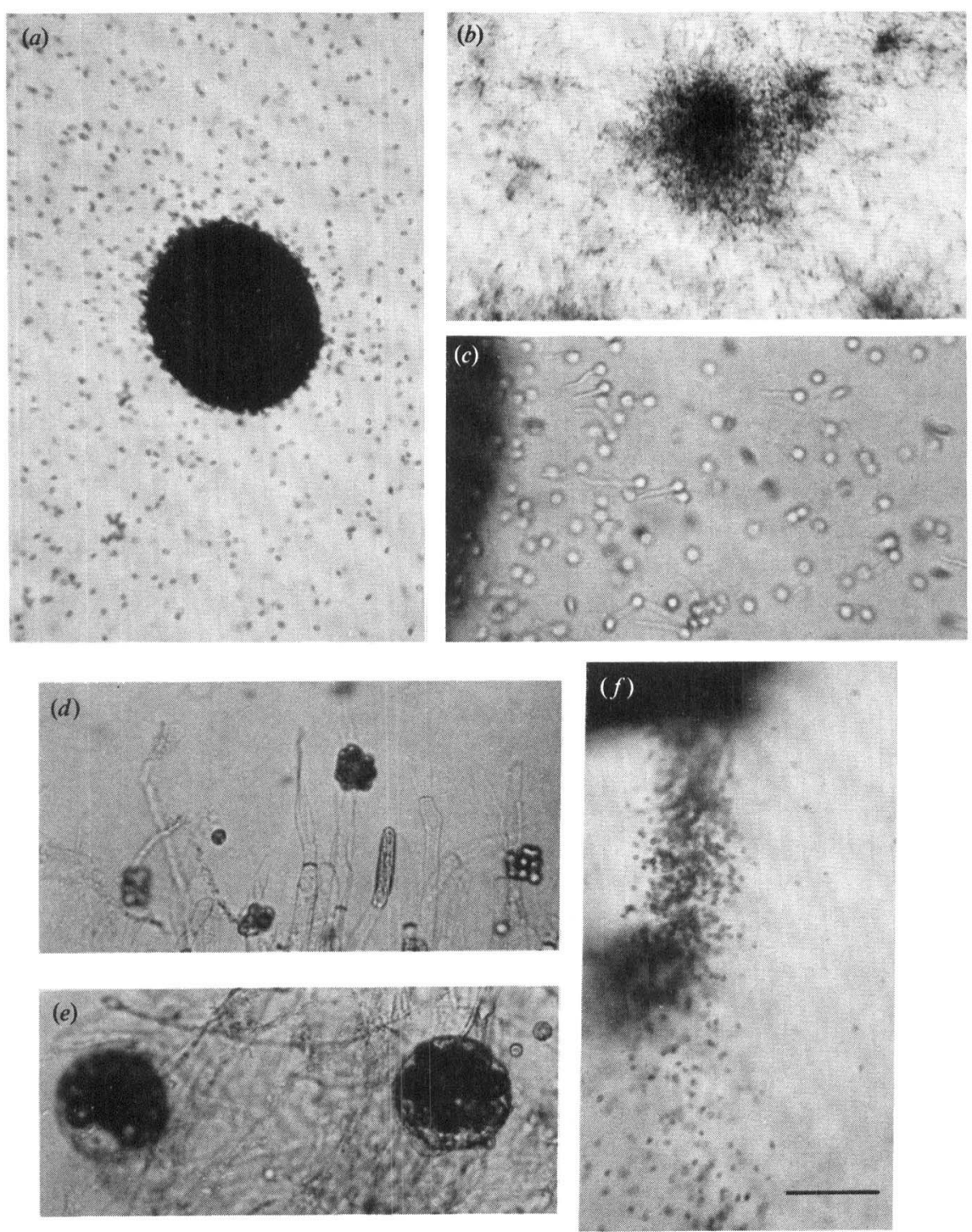

Fig. 1. Photomicrographs showing aspects of aggregation by Achlya spores. Spontaneous globular $(a)$ and plaque $(b)$ aggregates; the positive chemotropic response of germlings in response to a globular aggregate (located at left) (c); asexual sporangia with spore clusters $(d)$ and oogonia $(e)$ which developed from spore aggregates in the absence of exogenous nutrients. Portion of a zoospore swarm approaching the tip of a capillary tube (top) containing PYG agar $(f)$. Bar, $100 \mu \mathrm{m}$.

Table 1. Growth of spontaneous aggregates

For experiments 1 and 2 , values are the means $( \pm \operatorname{SEM})$ of 19 and 10 observations, respectively.

\begin{tabular}{cccccc}
\hline \hline Expt & $\begin{array}{c}\text { No. of } \\
\text { aggregates }\end{array}$ & $10^{-6} \times \begin{array}{c}\text { Aggregate vol. } \\
\left(\mu \mathrm{m}^{3}\right)\end{array}$ & $\begin{array}{c}\text { Time* } \\
(\mathrm{min})\end{array}$ & $\begin{array}{c}10^{-6} \times \text { Growth rate } \\
\left(\mu \mathrm{m}^{3} \mathrm{~min}^{-1}\right)\end{array}$ & $\begin{array}{c}10^{-3} \times \mathrm{Growth} \mathrm{rate}^{*} \\
\left(\mathrm{spores} \mathrm{min}^{-1}\right)\end{array}$ \\
\hline 1 & 19 & $47.4 \pm 31.9$ & $5.8 \pm 2.2$ & 8.2 & 3.9 \\
2 & 10 & $144.2 \pm 395 \cdot 1$ & $6.7 \pm 2.5$ & 21.5 & $10 \cdot 2$ \\
\hline \hline
\end{tabular}

* Time elapsed between initial measurement of aggregate and time when no further attraction of zoospores was observed.

† Growth rate assuming that spores represent $50 \%$ of the aggregate volume. 


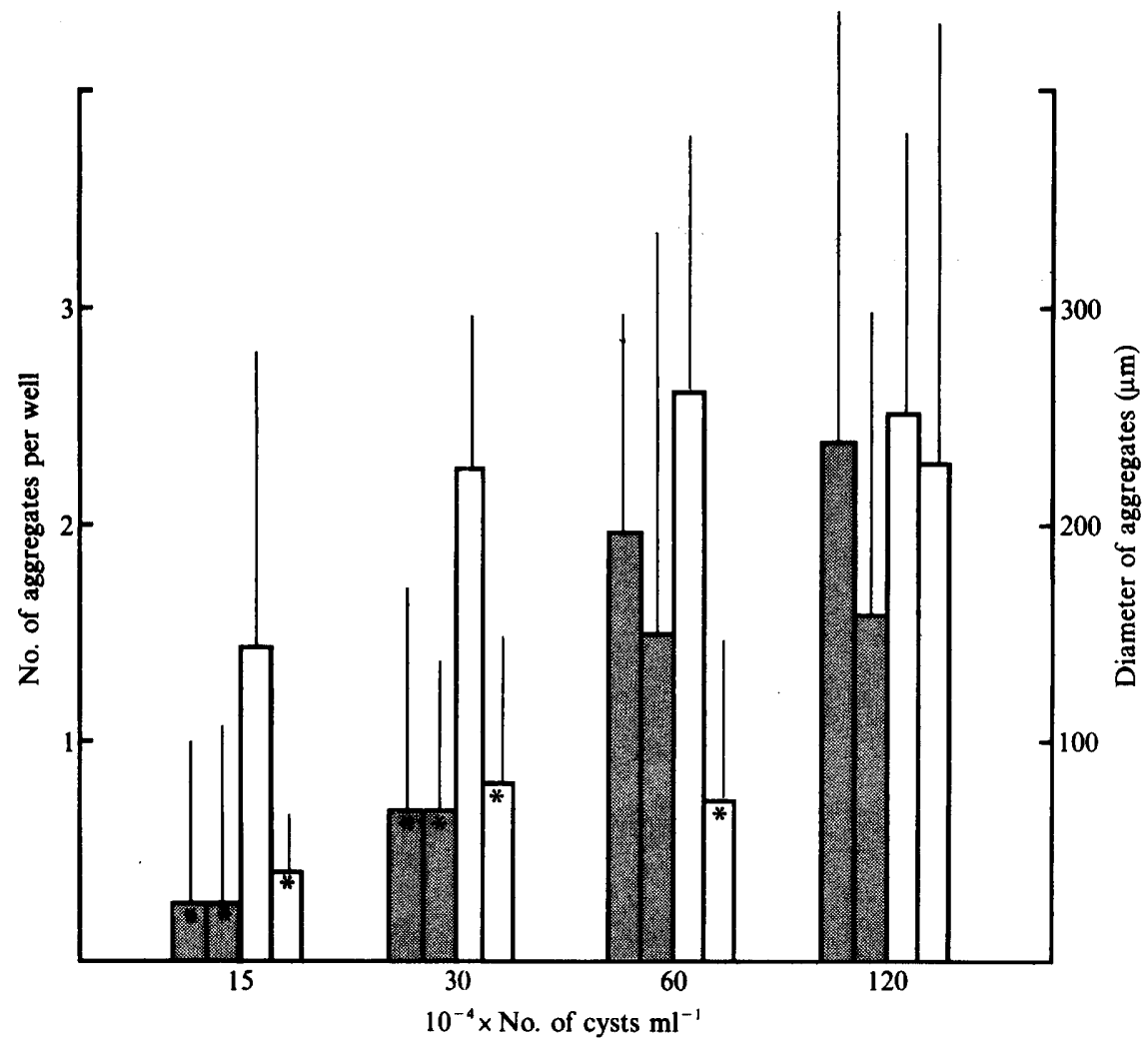

Fig. 2. Effect of cyst concentration on the aggregation of zoospores. The means of two experiments $(n=12$ for each) are shown. No of aggregates per well; $\square$, diameter of aggregates $(\mu \mathrm{m})$. The vertical bars represent $1 \times$ the standard error of each mean. Asterisks denote means significantly different from the controls $\left(120 \times 10^{-4}\right.$ cysts $\left.\mathrm{ml}^{-1}\right)$ at $P=0.05$ using Student's $t$-test.

Table 2. Chemotaxis to spore extracts

Values are the means $( \pm \mathrm{SEM})$ of three replicates.

\begin{tabular}{|c|c|c|}
\hline \multirow[b]{2}{*}{ Extract } & \multicolumn{2}{|c|}{ No. of spores per capillary tube } \\
\hline & Control & Test \\
\hline Cysts, $\mathrm{S}^{\dagger}$ & $10 \cdot 8 \pm 3 \cdot 3$ & $41 \cdot 7 \pm 7 \cdot 0^{* *}$ \\
\hline Zoospores, filtered & $10.8 \pm 3.3$ & $25.9 \pm 8.5^{*}$ \\
\hline Zoospores, vortexed for $5 \mathrm{~s}, \ddagger \mathrm{S}$ & $10 \cdot 8 \pm 3 \cdot 3$ & $43 \cdot 4 \pm 10 \cdot 7^{* *}$ \\
\hline Aggregating zoospores, $\mathrm{S}$ & $7 \cdot 2 \pm 4 \cdot 5$ & $32 \cdot 0 \pm 3 \cdot 2^{* *}$ \\
\hline Aggregating zoospores, S, heated $\S$ & $7 \cdot 2 \pm 4.5$ & $54 \cdot 8 \pm 24 \cdot 7^{* *}$ \\
\hline
\end{tabular}

${ }^{*},{ }^{* *}$, Significantly different from control at $P=0 \cdot 05$ and $0 \cdot 01$, respectively, using Student's $t$-test.

$+\mathrm{S}$, supernatant from centrifugation for $2 \mathrm{~min}$ at 14000 r.p.m.

$\ddagger$ Vortexing destroyed $33 \%$ of the spores.

$\S$ Supernatant was heated for $5 \mathrm{~min}$ in a water bath at $100{ }^{\circ} \mathrm{C}$.

While capillary tubes containing attractant solutions failed to induce aggregation, tubes loaded with agar media containing PYG or methionine at $2 \times 10^{-3} \mathrm{M}$, a concentration which we determined to be optimal for zoospore chemotaxis, induced rapid aggregation. In suspensions containing $10^{5}-10^{6}$ spores $\mathrm{ml}^{-1}$ a macroscopic globular aggregate formed at the tip of the capillary tube. Successive attempts, each using a fresh capillary loaded with PYG agar, at initiating aggregates in the same container of zoospores showed a progressive decrease in aggregate size and in the duration of the aggregation process (not shown). With the reduced zoospore numbers during each second and third attempt, it became apparent that the zoospores approached the 

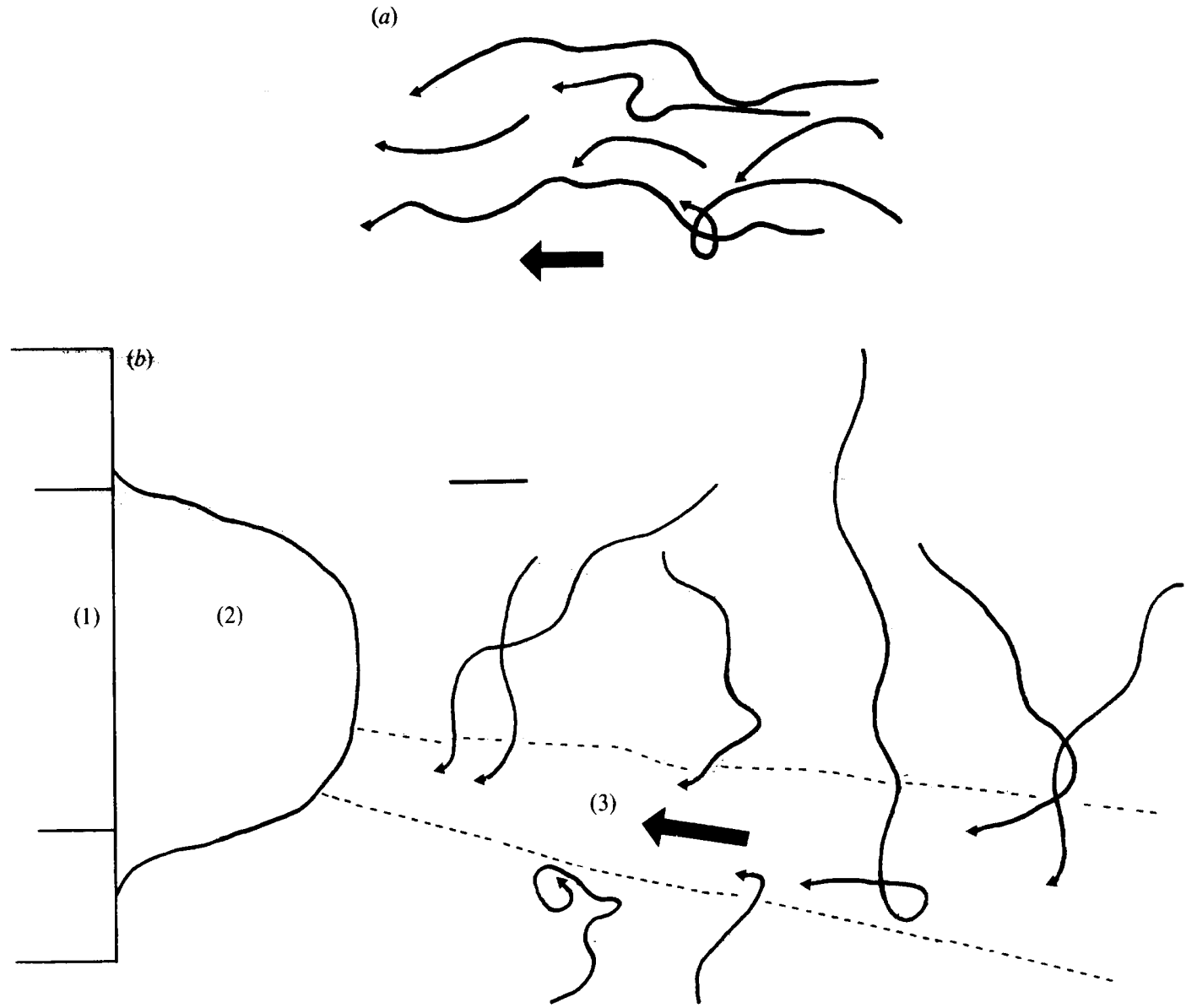

Fig. 3. Zoospore tracks plotted from video cassette recordings of spores aggregating in response to a capillary tube loaded with PYG agar: $(a)$ individual zoospores within a swarm maintained an almost constant orientation towards the attractant located in the direction of the large arrow; $(b)$ a plot showing a capillary tube tip (1), spore aggregate (2) and zoospore swarm (3) with tracks of zoospores recruited towards the swarm. Bar, $100 \mu \mathrm{m}$

Table 3. Effect of nutrient addition to the zoospore suspension on aggregation (duration and aggregate size) in response to a capillary tube containing PYG agar

Values are the means $( \pm$ SEM) of four (expt 1) or two (expts 2 and 3$)$ replicates.

\begin{tabular}{|c|c|c|c|c|}
\hline Expt & $\begin{array}{l}\text { No. of } \\
\text { replicates }\end{array}$ & Addition & $\begin{array}{c}\text { Duration of } \\
\text { aggregate } \\
\text { growth (min) }\end{array}$ & $\begin{array}{c}\text { Size of } \\
\text { aggregate }\left(\mathrm{mm}^{3}\right)\end{array}$ \\
\hline 1 & 4 & $\begin{array}{l}\text { CaMOPS (control) } \\
\text { Met, } 2 \times 10^{-3} \mathrm{M}\end{array}$ & $\begin{array}{l}31.7 \pm 1.5 \\
37.5 \pm 3.5^{*}\end{array}$ & $\begin{array}{l}0.56 \pm 0.24 \\
2.54 \pm 0.42^{* *}\end{array}$ \\
\hline 2 & 2 & $\begin{array}{l}\text { CaMOPS } \\
\text { Met, } 2 \times 10^{-3} \mathrm{M} \\
\text { PYG diluted } 1: 1000 \\
\text { PYG diluted } 1: 100 \\
\text { PYG diluted } 1: 10\end{array}$ & $\begin{aligned} 36.5 & \pm 2.1 \\
93.0 \pm 1.4^{* *} & \pm 2.8 \\
37.0 \pm & \pm 2.8^{*} \\
25.0 \pm & \pm 0.0^{* *}\end{aligned}$ & $\begin{array}{l}0.55 \pm 0.46 \\
1.20 \pm 0.36 \\
0.14 \pm 0.10 \\
0.02 \pm 0.01 \\
0.00 \pm 0.00\end{array}$ \\
\hline 3 & 2 & $\begin{array}{l}\text { CaMOPS } \\
\text { Met, } 2 \times 10^{-3} \mathrm{M} \\
\text { Ala, } 2 \times 10^{-3} \mathrm{M} \\
\text { PYG diluted } 1: 100\end{array}$ & $\begin{array}{r}31.0 \pm 1.4 \\
102.5 \pm 3.5^{* *} \\
91.0 \pm 15 \cdot 5^{*} \\
21.5 \pm 2.12^{*}\end{array}$ & $\begin{array}{l}0.22 \pm 0.19 \\
1.75 \pm 0.84 \\
1.05 \pm 0.95 \\
0.03 \pm 0.01\end{array}$ \\
\hline
\end{tabular}

*,**, Significantly different from control at $P=0.05$ and 0.01 , respectively, using Student's $t$-test. 
aggregate as a compact swarm over $2.5 \mathrm{~mm}$ long, and only $0 \cdot 2-0 \cdot 3 \mathrm{~mm}$ wide (Fig. $1 f$ ). Frame-by-frame analysis of videotape recordings showed that most zoospores in the swarm maintained an almost constant orientation towards the attractant (Fig. $3 a$ ), and that most zoospores recruited to the swarm responded to attractants from the swarm itself rather than from the capillary tube (Fig. $3 b$ ), consistent with the possibility that adelphochemotaxis might play a major role in chemotactically induced aggregation.

When aggregation was initiated by a PYG-agarloaded capillary tube, nutrients added to the zoospore suspension affected both the duration of the aggregation process and the size of the aggregate (Table 3). As expected, PYG diminished the duration of aggregation and tended to decrease aggregate size. However both Lmethionine and L-alanine, tested at a concentration optimal for chemotaxis, promoted aggregation in response to PYG agar.

\section{Discussion}

The growth rate of spontaneous aggregates of Achlya spores, estimated at 4-10 $\times 10^{3}$ spores $\mathrm{min}^{-1}$ (Table 1$)$ is probably one of the higher aggregation rates observed in motile eukaryotic cells.

Although 'aggregation' of the pattern swimming type has been suggested to be an in vitro artefact associated with unusually high concentrations of zoospores (Carlile, 1983), we consider that adelphotaxis and auto-aggregation may not require extraordinary zoospore concentrations. While macroscopic auto-aggregates are most conspicuous at high zoospore concentrations, aggregation at lower concentrations typically yields microscopic cyst plaques. Enhanced concentrations of zoospores may arise in nature as a result of chemotaxis to a substrate. Experimentally, aggregation can readily be initiated by providing a nucleation centre containing a chemotactic attractant such as PYG or methionine agar. In the past, such aggregation was interpreted solely as chemotaxis to an exogenous stimulus (Fischer \& Werner, 1958; Royle \& Hickman, 1964a). According to our two-stage model for chemotactic aggregation, the primary exogenous signal emanating from the substrate is reinforced by a secondary signal from the aggregating zoospores themselves. The secondary or adelphotactic signal may be based either on attractant 'leakage' from spores or on a chemotactic relay system analogous to that present in aggregating Dictyostelium amoebae (Alcantara \& Monk, 1974; Schaffer, 1975). Adelphotaxis in response to signals from zoospores attracted by an exogenous chemotactic signal is a potential means of amplifying an initial aggregation signal, and would help to explain the 'phenomenal accumulation' of zoospores observed in response to a wide range of attractant compounds (Hickman \& Ho, 1966, Royle \& Hickman, 1964a, b).

We confirmed the existence of a time-limit on autoaggregation reported by Lounsbury (1927), who commented that after aggregation ceased the remaining zoospores in the field ... exhibited no attraction to the newly formed spore ball'. We observed that exogenously induced aggregation shares this time-limited feature, and that the duration of aggregation could be modified by nutrients incorporated into the zoospore suspension, with methionine and alanine tending to increase both aggregation time and aggregate size. This unexpected response may enhance aggregation in the presence of natural substrates which release amino acids into the surrounding medium; such compounds might enhance aggregation even in the absence of an amino acid gradient. This reponse also disqualifies methionine and alanine as adelphotaxins.

Adelphotaxis and auto-aggregation of water mould spores may be viewed as anti-entropic mechanisms with a number of possible functions related to resource consolidation. The importance of inoculum potential on colonization reported in plant parasites with a zoosporic phase (MacWithey, 1965; Kraft \& Endo, 1966) is probably also applicable to colonization success in saprophytes. Both exogenous and adelphotactic signals may serve a sexual function in recruitment of potential mates to a single substrate. In such cases zoospores would have a paragametic role, and the adelphotaxin would serve as a non-mating-type-specific sexual pheromone. Finally, aggregate formation may permit an extra cycle of spore-production in the absence of exogenous nutrients.

Adelphotaxis as described by Lounsbury (1927) was a rare event absent in thousands of water mould cultures examined, observed only over a limited time, and apparently not readily repeatable. We expect adelphotaxis to be a feature of motile behaviour in additional genera of zoosporic fungi and suggest that it may be a component of the chemotactic response of other motile cells.

We thank the National Science and Engineering Research Council of Canada for financial support, and Usha Jacob for technical assistance.

\section{References}

AlCaNTARA, F. \& MoNk, M. (1974). Signal propagation during aggregation in the slime mould Dictyostelium discoideum. Journal of General Microbiology 85, 321-334.

CANTINo, E. C. \& LOVETT, J. S. (1960). Respiration of Blastocladiella during bicarbonate-induced morphogenesis in synchronous culture. Physiologia Plantarum 13, 450-458. 
CARLILE, M. J. (1983). Motility, taxis and tropism in Phytophthora. In Phytophthora, Its Biology, Taxonomy, Ecology and Pathology, pp. 95107. Edited by D. C. Erwin, S. Bartnicki-Garcia \& P. H. Tsao. St Paul, Minnesota: American Phytopathological Society.

Fischer, F. G. \& WeRneR, G. (1958). Die Chemotaxis der Schwarmsporen von Wasserpilzen (Saprolegniaceen). Zeitschrift für Physiologische Chemie 310, 65-91.

HARTOG, M. M. (1888). Adelphotaxy, an undescribed form of irritability. Reports of the British Association for the Advancement of Science 1888, 72.

HICKMAN, C. J. \& Ho, H. H. (1966). Behaviour of zoospores in plant pathogenic Phycomycetes. Annual Review of Phytopathology 4, 195220.

JAFFE, L. F. (1966). On autotropism in Botrytis: measurement technique and control by $\mathrm{CO}_{2}$. Plant Physiology 41, 303-306.

Ko, W. H. \& Chase, L. L. (1973). Aggregation of zoospores of Phytophthora palmivora. Journal of General Microbiology 78, 79-82.

Ko, W. H., Chase, L. L. \& Kunimoto, R. K. (1973). A microsyringe method for determining concentration of fungal propagules. Phytopathology 63, 1206-1207.

KRAFT, J. M. \& ENDO, R. M. (1966). Zoospore infection of bentgrass roots by Pythium aphanidermatum. Phytopathology 56, 149.

LECHMERE, A. E. (1910). An investigation of a species of Saprolegnia. New Phytologist 9, 305-319.

LOUNSBURY, J. A. (1927). A case of phenomenal zoospore behaviour of an apparently sterile Isoachlya and a description of the plant. Transactions of the Wisconsin Academy 23, 539-549.

MACWITHEY, H. S. (1965). Factors affecting the prevalence of black root disease of sugar beets. Phytopathology 55, 1066.

Manavathu, E. K. \& Thomas, D. D. (1985). Chemotropism of Achlya ambisexualis to methionine and methionyl compounds. Journal of General Microbiology 131, 751-756.

Musgrave, A., Ero, L., Scheffer, R. \& Oehlers, E. (1977). Chemotropism of Achlya bisexualis germ hyphae to casein hydrolysate and amino acids. Journal of General Microbiology 101, 65-70.

Plesset, M. S., Whipple, C. G. \& Winet, H. (1975). Analysis of the steady state of bioconvection in swarms of swimming microorganisms. In Swimming and Flying in Nature, vol. 1, pp. 339-360. Edited by T. Y.-T. Wu, C. J. Brokaw \& C. Brennan. New York: Plenum Press.

PORTER, J. R. \& SHAw, D. S. (1978). Aggregation of Phytophthora drechsleri zoospores: pattern analysis suggests a taxis. Transactions of the British Mycological Society 71, 515-519.

Robinson, P. M. (1973). Chemotropism in fungi. Transactions of the British Mycological Society 61, 303-313.

RoYLE, D. J. \& HiCkMAN, C. J. (1964a). Analysis of factors governing in vitro accumulation of zoospores of Pythium aphanidermatum on roots. I. Behaviour of zoospores. Canadian Journal of Microbiology 10, 151-162.

Royle, D. J. \& Hickman, C. J. (1964b). Analysis of factors governing in vitro accumulation of zoospores of Pythium aphanidermatum on roots. II. Substances causing the response. Canadian Journal of Microbiology 10, 201-219.

SCHAFFER, B. M. (1975). Secretion of cyclic AMP induced by cyclic AMP in the cellular slime mould Dictyostelium discoideum. Nature, London 255, 549-552.

THOMAS, D. D. (1989). A method for preparing Achlya cysts with high zoosporogenic potential. Mycological Research 92, 233-235.

ZentMYer, G. A. (1961). Chemotaxis of zoospores for root exudates. Science 133, 1595-1596. 